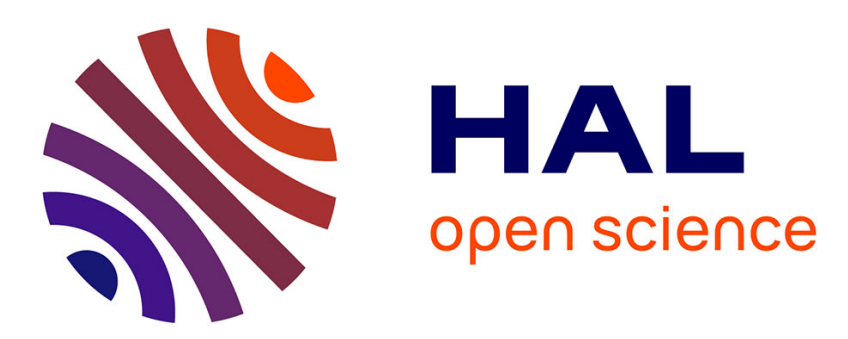

\title{
Antenna Array Design for Music in The Presence of Spatially Distributed Sources
}

Wenmeng Xiong, José Picheral, Sylvie Marcos

\section{To cite this version:}

Wenmeng Xiong, José Picheral, Sylvie Marcos. Antenna Array Design for Music in The Presence of Spatially Distributed Sources. 23rd European Signal Processing Conference (EUSIPCO 2015), Aug 2015, Nice, France. hal-01235084

\section{HAL Id: hal-01235084 \\ https://hal.science/hal-01235084}

Submitted on 3 Dec 2015

HAL is a multi-disciplinary open access archive for the deposit and dissemination of scientific research documents, whether they are published or not. The documents may come from teaching and research institutions in France or abroad, or from public or private research centers.
L'archive ouverte pluridisciplinaire HAL, est destinée au dépôt et à la diffusion de documents scientifiques de niveau recherche, publiés ou non, émanant des établissements d'enseignement et de recherche français ou étrangers, des laboratoires publics ou privés. 


\title{
ANTENNA ARRAY DESIGN FOR MUSIC IN THE PRESENCE OF SPATIALLY DISTRIBUTED SOURCES
}

\author{
Wenmeng XIONG, José PICHERAL, Sylvie MARCOS \\ Laboratoire des signaux et systèmes, CentraleSupélec-CNRS-Université Paris-Sud, \\ 91192 Gif-sur-Yvette,France
}

\begin{abstract}
In this paper, the impact of array geometry on the direction of arrival (DOA) estimation of spatially distributed sources impinging on a sensor array is considered. Taking into account the coherently distributed source model proposed in [1], we establish closed-form expressions of the MUSIC-based DOA estimation error as functions of the positions of the array sensors in the presence of model errors due to the angular dispersion of the signal sources. The impact of the array geometry is studied and particular array designs are proposed to make DOA estimation more robust to source dispersion. The analytical results are validated by numerical simulations.
\end{abstract}

Index Terms - array signal processing, distributed sources, array geometry, performance, MUSIC

\section{INTRODUCTION}

The direction of arrival (DOA) estimation based on snapshots received on a sensor array has been widely studied with plenty of methods [2]. Among these methods, the multiple signal classification (MUSIC) [3] is famous for its high resolution in the case of punctual sources. However, in many applications, such as acoustic source imaging [4], the physical sources can no longer be considered as point sources and a spatially distributed model of the sources could be more appropriate, which degrades the performance of the DOA estimation obtained by MUSIC.

The models for spatially distributed sources have been classified into two types, namely incoherently distributed (ID) sources and coherently distributed (CD) sources. On one hand, for ID sources, signals coming from different points of the same distributed source can be considered uncorrelated. On the other hand, in the scenario of CD sources, the received signal components are delayed and scaled replicas from different points of the same one [1]. For CD sources, the performances of MUSIC with discretely distributed sources and continuously distributed sources have been investigated in [5], and [6], respectively. As expected, the mismatch due to the angular dispersion between the steering vector model of MUSIC and the actual steering vectors of the sources causes estimation errors. MUSIC-based methods for the joint esti- mation of both the DOA and the angular dispersion parameter can be used to avoid the angular dispersion mismatch problem [1]. More recently, another MUSIC-based method [7] makes it possible to have a good performance regardless of the angular distribution shape of the signal sources.

The array geometry on the DOA estimation has been studied in plenty of publications. Optimal array geometries have been designed to reach isotropic and/or optimal performance based on the Cramér-Rao bound (CRB) criterion (eg : [8, 9]). More recently, based on the spatial aliasing phenomenon, a class of non-uniform array geometries composed of two or more uniform linear arrays (ULAs) with different interelement spacing has been used to reduce the computational burden of Maximum Likelihood (ML) estimator [10].

In this paper, we focus on the impact of the array geometry on the performance of the MUSIC estimator with the CD source model, in the presence of model errors due to the angular dispersion of the source signals. Based on the work in [6], we first propose an analytical expression of the DOA estimation error as an explicit function of the sensor positions for a single source. Subsequently, the array design of special geometries to ensure robustness to the angular dispersion of the signal sources will be investigated. In the case of two sources, the DOA estimation error with a uniform circular array (UCA) will be proved to be an explicit function of the DOA separation between the two sources. Simulation results illustrate the validation of the theoretical results.

The organization of this paper is as follows. The signal model and a brief recall of MUSIC are given in section 2 . The particular array geometry design is investigated in section 3. Numerical simulations are presented in section 4 to validate the analytical results of the previous section. Finally, conclusions are given in section 5 .

\section{SIGNAL MODEL AND MUSIC ESTIMATOR}

Let us consider $q$ spatially CD far-field narrow-band sources impinging on an array of $M$ sensors. The sources arrive from the DOA $\theta_{1}, \ldots, \theta_{q}$, and the position of the $m-t h$ sensor is given by the polar coordinate $\rho_{m}$ and $\alpha_{m}$. Without loss of generality, the signals and the sensors are assumed to be in the same plane, as shown in Figure 1a. The $q$ 
source signals and the $M$ signals received by the array at moment $t$ are denoted by $\mathbf{s}(t)=\left[s_{1}(t), \ldots, s_{q}(t)\right]^{T}$ and $\mathbf{y}(t)=\left[y_{1}(t), \ldots, y_{M}(t)\right]^{T}$, respectively, with:

$$
\mathbf{y}(t)=\mathbf{C}(\theta) \mathbf{s}(t)+\mathbf{n}(t),
$$

where $\mathbf{n}(t) \in \mathbb{C}^{M \times 1}$ represents the complex Gaussian distributed additive noise, $\mathbf{C}(\theta)=\left[\mathbf{c}_{h_{1}}\left(\theta_{1}\right), \ldots, \mathbf{c}_{h_{q}}\left(\theta_{q}\right)\right] \in$ $\mathbb{C}^{M \times q}$ is the array steering matrix composed of $q$ steering vectors $\mathbf{c}_{h i}(\theta)$ that can be written as proposed in [1] by:

$$
\mathbf{c}_{h_{i}}\left(\theta_{i}\right)=\int_{-\frac{\pi}{2}}^{\frac{\pi}{2}} \mathbf{a}\left(\theta_{i}+\phi\right) h_{i}(\phi) d \phi,
$$

where $i=1 \ldots q$, and $\mathbf{a}(\theta)$ is the steering vector for a point source, which can be given by:

$$
\mathbf{a}\left(\theta_{i}\right)=\left[e^{-j 2 \pi \frac{\rho_{1}}{\lambda} \cos \left(\theta_{i}-\alpha_{1}\right)}, \ldots, e^{-j 2 \pi \frac{\rho_{M}}{\lambda} \cos \left(\theta_{i}-\alpha_{M}\right)}\right]^{T}
$$

where $\lambda$ is the wavelength, and $[\cdot]^{T}$ is the transpose operation.

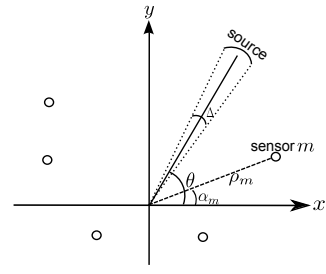

(a) Planar array and source DOAs

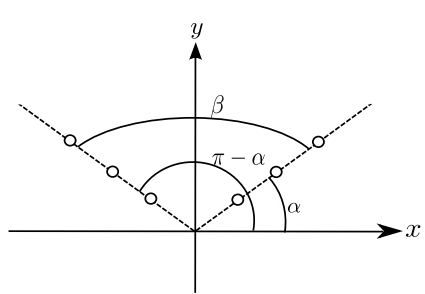

(b) V-shape array
Fig. 1: Illustration of planar arrays

The function $h(\phi)$ is introduced to describe the angular spread distribution (for instance, Uniform and Gaussian distributions) and it can be parameterized by an angular dispersion $\Delta$ which is omitted in the notation. The source signals and the additive noise are considered to be complex centered Gaussian independent random variables. Assuming that signals and noises are uncorrelated and the sources are uncorrelated with each other, the correlation matrix is given by:

$$
\mathbf{R}=E\left[\mathbf{y} \mathbf{y}^{H}\right]=\mathbf{C} \mathbf{R}_{s} \mathbf{C}^{H}+\sigma_{b}^{2} \mathbf{I},
$$

where $E[$.$] is the expectation operator, \mathbf{R}_{s}$ and $\sigma_{b}^{2}$ are the source covariance matrix and the noise variance, respectively.

Under the hypothesis that $q<M$ and $\mathbf{R}_{s}$ and $\mathbf{C}$ are not rank deficient, it is well known that the decomposition of $\mathbf{R}$ into eigenvalues $\lambda_{m}$ and eigenvectors $\mathbf{e}_{m}$ is as follows :

$$
\mathbf{R}=\sum_{m=1}^{M} \lambda_{m} \mathbf{e}_{m} \mathbf{e}_{m}=\mathbf{U} \Lambda_{s} \mathbf{U}^{H}+\sigma_{b}^{2} \mathbf{V} \mathbf{V}^{H},
$$

where $\mathbf{U}=\left[\mathbf{e}_{1}, \ldots, \mathbf{e}_{q}\right]$ spans the signal subspace defined by the columns of $\mathbf{C}$ and $\mathbf{V}=\left[\mathbf{e}_{q+1}, \ldots, \mathbf{e}_{M}\right]$ spans the noise subspace defined as the orthogonal complement of $\mathbf{U}, \Lambda_{s}=$ $\operatorname{diag}\left\{\lambda_{1}, \ldots, \lambda_{q}\right\}$.
The MUSIC method makes use of the orthogonal property of subspaces spanned by $\mathbf{C}(\theta)$ and $\mathbf{V}$ to estimate the DOA $\theta$. In practice it is difficult to know exactly the angular dispersion of the actual sources, consequently, the steering vector model of the punctual source $\mathbf{a}(\theta)$ is used here instead of $\mathbf{c}(\theta)$ to estimate the value of $\theta$ so as to have:

$$
\hat{\theta}_{i}=\underset{\theta}{\operatorname{argmax}} \frac{1}{\left\|\mathbf{a}^{H}(\theta) \mathbf{V}\right\|^{2}} .
$$

We note $\Pi=\mathbf{V V}^{H}$ the noise subspace projector corresponding to the actual sources, and $\tilde{\Pi}=\mathbf{I}-\mathbf{A}\left(\mathbf{A}^{H} \mathbf{A}\right)^{-1} \mathbf{A}^{H}$ the noise subspace projector corresponding to the model in the estimator, with $\mathbf{I}$ the $M \times M$ identity matrix, $\mathbf{A}=$ $\left[\mathbf{a}\left(\theta_{1}\right), \ldots, \mathbf{a}\left(\theta_{q}\right)\right]$. Due to the mismatch of the angular dispersion parameter between the model of the MUSIC estimator $\mathbf{a}(\theta)$ and the actual source $\mathbf{c}_{h_{i}}\left(\theta_{i}\right)$, and the fact that the number of snapshots can not be infinite in practice, an estimation error then arises [6]. In this paper, we focus on the influence of the antenna array geometry on the DOA estimation error due to the model error, the covariance matrix is thus assumed to be perfectly known. In the following, for simplicity we note $\mathbf{c}_{i}$ for $\mathbf{c}_{h_{i}}\left(\theta_{i}\right)$, and $\mathbf{a}_{i}$ for $\mathbf{a}\left(\theta_{i}\right)$, respectively. For source $i$, from eq(13) in [6], the DOA estimation error can be given by:

$$
\Delta \theta_{i}=\hat{\theta}_{i}-\theta_{i}=\frac{\mathcal{R} e\left\{\dot{\mathbf{a}}_{i}^{H} \tilde{\Pi} \mathbf{c}_{i}\right\}}{\dot{\mathbf{a}}_{i}^{H} \tilde{\Pi} \dot{\mathbf{a}}_{i}} .
$$

where $\dot{\mathbf{a}}_{i}=\frac{\partial \mathbf{a}}{\partial \theta}$.

\section{INFLUENCE OF THE ANTENNA ARRAY GEOMETRY}

In the following, we show that $\Delta \theta_{i}$ can be expressed as an explicit function of the antenna positions, the effects of special arrays can thus be investigated.

\subsection{Single source}

In this subsection, we study the particular scenario where there is only one source arriving from the DOA $\theta$. Notice that in this case $\tilde{\Pi}=\mathbf{I}-\frac{1}{M} \mathbf{a a}^{H}$, the DOA estimation error can then be given by:

$$
\Delta \theta=\frac{\mathcal{R} e\left\{\dot{\mathbf{a}}^{H} \mathbf{c}-\frac{1}{M} \dot{\mathbf{a}}^{H} \mathbf{a} \mathbf{a}^{H} \mathbf{c}\right\}}{\dot{\mathbf{a}}^{H} \dot{\mathbf{a}}-\frac{1}{M}\left|\dot{\mathbf{a}}^{H} \mathbf{a}\right|^{2}} .
$$

For the $m-t h$ sensor, let us define:

$$
\begin{aligned}
\varphi_{m} & =2 \pi \frac{\rho_{m}}{\lambda} \cos \left(\theta-\alpha_{m}\right), \\
\dot{\varphi}_{m} & =-2 \pi \frac{\rho_{m}}{\lambda} \sin \left(\theta-\alpha_{m}\right), \\
u_{m} & =\int \cos \left(\varphi_{m}(\theta+\phi)-\varphi_{m}(\theta)\right) h(\phi) d \phi, \\
v_{m} & =\int \sin \left(\varphi_{m}(\theta+\phi)-\varphi_{m}(\theta)\right) h(\phi) d \phi .
\end{aligned}
$$


Introducing the notations defined in (9) in (8), $\Delta \theta$ can be given by:

$$
\Delta \theta=\frac{\overline{\dot{\varphi} \cdot v}-\overline{\dot{\varphi}} \cdot \bar{v}}{\overline{\dot{\varphi}^{2}}-(\overline{\dot{\varphi}})^{2}}
$$

where $\bar{x}=\frac{1}{M} \sum_{m=1}^{M} x_{m}$ is the mean value operator for the variable $x$. Eq (10) makes it possible to design the geometry in order to minimize $\Delta \theta$,

Assuming that the angular dispersion of the distributed source is small enough with a symmetrical distribution, we introduce a third order Taylor approximation in $\phi$ of $\varphi_{m}(\theta+$ $\phi)-\varphi_{m}(\theta)$ so as:

$$
v_{m} \approx \frac{1}{2} \ddot{\varphi}_{m} \sigma^{2},
$$

where $\ddot{\varphi}_{m}(\theta)=-2 \pi \frac{\rho_{m}}{\lambda} \cos \left(\theta-\alpha_{m}\right)$, and $\sigma^{2} \triangleq \int \phi^{2} h(\phi) d \phi$ is the parameter related to the angular dispersion of the source signal.

Let us introduce (11) in (10), $\Delta \theta$ can be given by:

$$
\Delta \theta=\frac{1}{2} \sigma^{2} \frac{\overline{\dot{\varphi} \cdot \ddot{\varphi}}-\overline{\dot{\varphi}} \cdot \bar{\varphi}}{\overline{\dot{\varphi}^{2}}-(\overline{\dot{\varphi}})^{2}} .
$$

We can observe that this approximative expression of the DOA estimation error is proportional to the angular dispersion of the signal source.

\subsubsection{Uniform circular array}

Inspired by (12), we find that for a UCA with an even number of symmetrical elements, where $\rho_{m}=\rho$, and $\alpha_{m}=2 \pi \frac{(m-1)}{M}$ with $M$ even, the terms in the numerator can be null:

$$
\begin{aligned}
\overline{\dot{\varphi}} & =-\frac{1}{M} 2 \pi \frac{\rho}{\lambda} \sum_{m=1}^{M} \sin \left(\theta-\alpha_{m}\right) \\
& =-\frac{1}{M} 2 \pi \frac{\rho}{\lambda}\left[\sum_{m=1}^{\frac{M}{2}} \sin \left(\theta-\alpha_{m}\right)+\sum_{m=1}^{\frac{M}{2}} \sin \left(\theta-\alpha_{m}+\pi\right)\right] \\
& =0
\end{aligned}
$$

and similarly :

$$
\overline{\dot{\varphi} \cdot \ddot{\varphi}}=\frac{\left(2 \pi \frac{\rho}{\lambda}\right)^{2}}{M} \sum_{m=1}^{M} \underbrace{\sin \left(\theta-\alpha_{m}\right) \cos \left(\theta-\alpha_{m}\right)}_{\frac{1}{2} \sin \left(2 \theta-2 \alpha_{m}\right)}=0,
$$

which leads $\Delta \theta$ to be 0 , The result illustrates that a symmetrical even elements UCA can be robust to the model error due to the source dispersion, in the scenario of one source.

\subsubsection{V-shape array}

The V-shape array (VA) is another type of particular geometries which has been widely studied, thanks to its easiness to parameterize. It has been proven in [8] that with the array angle fixed to a specific value, a VA with any size can have an isotropic behavior, and outperforms the UCA in terms of CRB.

Let us assume that, without loss of generality, the two identical branches are symmetrical with respect to the $y$ axis, with no sensor placed at the origin, as depicted in Figure 1b, and that, the distance between two sensors is fixed to $d$. The coordinates of the sensors can be expressed as:

$$
\rho_{m}=\left\{\begin{array}{ll}
m d, & 1 \leqslant m \leqslant \frac{M}{2} \\
\left(m-\frac{M}{2}\right) d, & \frac{M}{2} \leqslant m \leqslant M
\end{array},\right.
$$

and:

$$
\alpha_{m}=\left\{\begin{array}{lr}
\alpha, & 1 \leqslant m \leqslant \frac{M}{2} \\
\pi-\alpha, & \frac{M}{2} \leqslant m \leqslant M
\end{array},\right.
$$

respectively.

Considering the described V-shape geometry, (12) can be derived as:

$\Delta \theta=\frac{1}{2} \sigma^{2} \frac{\sin \theta \cos \theta\left[(4+4 M) \cos ^{2} \alpha+(2-M) \sin ^{2} \alpha\right]}{(4+4 M) \cos ^{2} \alpha \sin ^{2} \theta+(2-M) \sin ^{2} \alpha \cos ^{2} \theta}$,

we can see that the DOA estimation error is independent of $d$.

To make $\Delta \theta$ equal to 0 , the numerator of (17) should be 0 , which yields:

$$
\tan ^{2} \alpha=\frac{4 M+4}{M-2},
$$

the expression reveals that the array angular $\alpha$ can be fixed to a special value to be robust to the model error due to the source dispersion, which depends roughly on the array size, and is robust to other parameters.

\subsubsection{Linear array}

Notice that the scenario of $\alpha_{m}=0$ in (12) corresponds to the linear array (LA), after some straightforward calculus from (12) with $\alpha_{m}=0$, the DOA estimation error is derived as:

$$
\Delta \theta=\frac{1}{2} \sigma^{2} \cot \theta
$$

which reveals that in the case of LA, the DOA estimation error does not depend on the sensors positions but only on the DOA and the model error. Taking into account that the model error can not be modified, the sensor array should be rotated to reduce the value of $\cot \theta$, so as to get a smaller estimation error, if $\theta$ is roughly known.

\subsection{Two sources in the case of a UCA}

In the case of multiple sources, we take the example of two sources arriving from $\theta_{i}$, where $i=1,2$ and assuming that $\theta_{2}>\theta_{1}$. For the $m-t h$ sensor, let us introduce the notation:

$$
\Delta \varphi_{m}=\varphi_{m}\left(\theta_{2}\right)-\varphi_{m}\left(\theta_{1}\right)=\varphi_{m, 2}-\varphi_{m, 1} .
$$


We here focus on the above-mentioned case of a UCA with an even number of symmetrically positioned sensors, which is easier to manipulate mathematically comparing to other geometries. Notice that with such a geometry:

$$
\mathcal{R} e\left\{\dot{\mathbf{a}}_{i}^{H} \mathbf{a}_{i}\right\}=-\sum_{m=1}^{M} 2 \pi \frac{\rho}{\lambda} \sin \left(\theta-\alpha_{m}\right)=0,
$$

and:

$\mathcal{R} e\left\{\dot{\mathbf{a}}_{i}^{H} \mathbf{c}_{i}\right\}=\sum_{m=1}^{M} \dot{\varphi}_{m, i} u_{m, i}=\underbrace{\left[\sum_{m=1}^{M / 2} \Phi\left(\theta_{i}\right)+\sum_{m=1}^{M / 2} \Phi\left(\theta_{i}+\pi\right)\right]}_{=0,}$

where $\Phi\left(\theta_{i}\right)=\int \dot{\varphi}_{m}\left(\theta_{i}\right)\left[\cos \left(\varphi_{m}\left(\theta_{i}+\phi\right)\right) \cos \left(\varphi_{m}\left(\theta_{i}\right)\right)+\right.$ $\left.\sin \left(\varphi_{m}\left(\theta_{i}+\phi\right)\right) \sin \left(\varphi_{m}\left(\theta_{i}\right)\right)\right] h(\phi) d \phi$.

Introducing (21), (22), and the expression $\mathbf{A}=\left[\mathbf{a}_{1}, \mathbf{a}_{2}\right]$ in (7) gives:

$$
\Delta \theta_{1}=\frac{\mathcal{R} e\left\{-M \dot{\mathbf{a}}_{1}^{H} \mathbf{a}_{2} \mathbf{a}_{2}^{H} \mathbf{c}_{1}+\dot{\mathbf{a}}_{1}^{H} \mathbf{a}_{2} \mathbf{a}_{1}^{H} \mathbf{c}_{1} \mathbf{a}_{2}^{H} \mathbf{a}_{1}\right\}}{\dot{\mathbf{a}}_{1}^{H} \dot{\mathbf{a}}_{1}\left(M^{2}-\left|\mathbf{a}_{2}^{H} \mathbf{a}_{1}\right|^{2}\right)-M\left|\dot{\mathbf{a}}_{1}^{H} \mathbf{a}_{2}\right|^{2}} .
$$

Introducing the notations of (9) and (20) in (23). The DOA estimation error as an explicit function of the sensor positions can be given by:

$$
\Delta \theta_{1}=\frac{\overline{\dot{\varphi}_{1} \cdot \sin (\Delta \varphi)}[\overline{\cos (\Delta \varphi)} \cdot \bar{u}-\overline{\cos (\Delta \varphi) \cdot u}]}{\overline{\dot{\varphi}_{1}^{2}}-\overline{\dot{\varphi}_{1}^{2}} \cdot \overline{\cos ^{2}(\Delta \varphi)}-\left(\overline{\dot{\varphi}_{1} \sin (\Delta \varphi)}\right)^{2}} .
$$

Let us note $\delta=\theta_{2}-\theta_{1}$, and introduce a third order approximation in $\delta$ in (24), after some straightforward calculus, the expression of the DOA estimation error for $\theta_{1}$ can be derived as:

$$
\Delta \theta_{1}=\frac{\left(18 \overline{\dot{\varphi}^{2}}-3 \delta^{2} \overline{\dot{\varphi}^{2}+\dot{\varphi}^{4}}\right)\left(\overline{\dot{\varphi}_{1}^{2} \cdot u_{1}}-\overline{\dot{\varphi}_{1}^{2}} \cdot \overline{u_{1}}\right)}{\delta\left[-9\left(\overline{\dot{\varphi}_{1}^{2}}\right)^{3}+12 \overline{\dot{\varphi}^{2}} \cdot \overline{\dot{\varphi}_{1}^{4}+\dot{\varphi}_{1}^{2}}-\delta^{2}\left(\overline{\dot{\varphi}_{1}^{2}+\dot{\varphi}_{1}^{4}}\right)^{2}\right]} .
$$

Let us consider the sign of $\Delta \theta_{1}$ : firstly, $\tilde{\Pi}$ is Hermitian, so the denominator $\dot{\mathbf{a}}_{i}^{H} \tilde{\Pi} \dot{\mathbf{a}}_{i}$ in (7) is non-negative, it is enough to focus on the numerator in (7) for $\theta_{1}$ which yields $\delta^{3}\left(18 \dot{\varphi}^{2}-\right.$ $\left.3 \delta^{2} \overline{\dot{\varphi}^{2}+\dot{\varphi}^{4}}\right)\left(\overline{\dot{\varphi}_{1}^{2} \cdot u_{1}}-\overline{\dot{\varphi}_{1}^{2}} \cdot \overline{u_{1}}\right)$ after some straightforward calculus; secondly with a small $\delta, 18 \overline{\dot{\varphi}^{2}}-3 \delta^{2} \overline{\dot{\varphi}^{2}+\dot{\varphi}^{4}}$ can be approximated by $18 \overline{\dot{\varphi}^{2}}$ which is positive; thirdly, by the CauchySchwarz inequality, $\overline{\dot{\varphi}_{1}^{2} \cdot u_{1}}-\overline{\dot{\varphi}_{1}^{2}} \cdot \overline{u_{1}}$ is negative. Therefore, $\Delta \theta_{1}$ is opposite to the sign of $\delta$.

For $\theta_{2}$, after some similar calculus, we have:

$$
\Delta \theta_{2}=-\Delta \theta_{1}
$$

It is interesting to see that, the value of the estimation error for both the two sources are inversely proportional to the value of $\delta$ for small $\delta$, which illustrates that the estimation error decreases as the sources move away from each other. Besides, $\Delta \theta_{1}$ is negative and $\Delta \theta_{2}$ is positive, which implies that when the two sources are close to each other, MUSIC is always able to give the estimation of two DOAs separately, despite that the value of the estimation error increases.

\section{NUMERICAL SIMULATIONS}

In this section, we validate the theoretical results through numerical experiments. In all simulations, uniformly distributed sources with the angular dispersion $\Delta_{0}=10^{\circ}$ are considered, the theoretical covariance matrix is assumed to be known, with $S N R=10 d B$. All the arrays are composed of $M=20$ sensors, the sensors are uniformly distributed on a circle with the diameter equal to $5 \lambda$ for the UCA, for the VA, 10 sensors are identically uniformly placed at the two branches, spaced by $d=\lambda / 4$, and the VA with $\alpha=0$ is considered as the case of LA. Such array configurations ensure that the UCA and the LA have the same horizontal aperture. The snapshots number $N=1000$ and 100 experiments are realized for the simulation results.

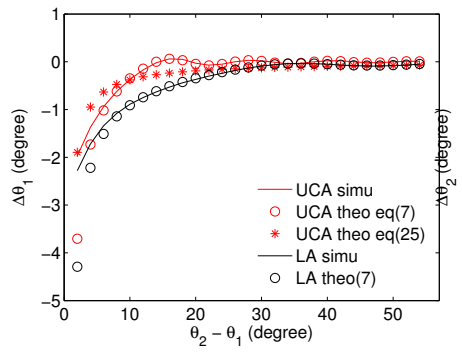

(a) $\theta_{1}$

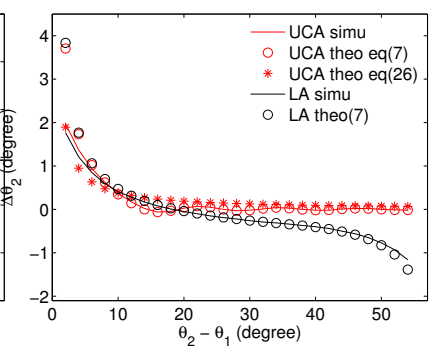

(b) $\theta_{2}$
Fig. 2: DOA estimation error vs. DOA separation (UCA with $M=20$, LA with $M=20$ and $d=\lambda / 4$, two sources with uniform angular dispersion, $\Delta_{0}=10^{\circ}, \theta_{c}=\frac{1}{2}\left(\theta_{1}+\theta_{2}\right)=$ $\left.60^{\circ}\right)$

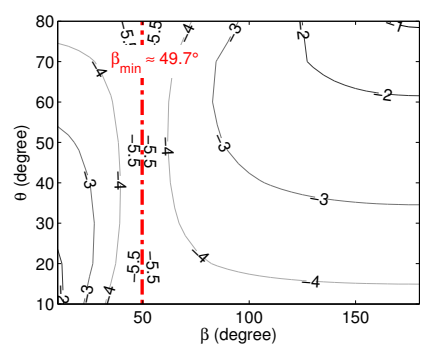

(a) theory $\ln |\Delta \theta|$ in eq(17)

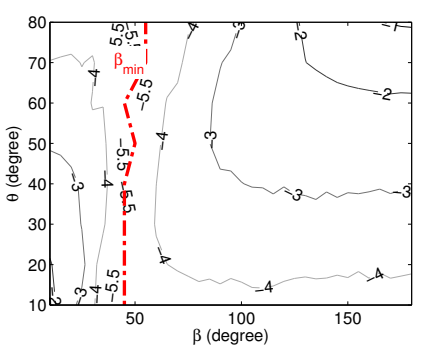

(b) simulation $\ln |\Delta \theta|$
Fig. 3: The absolute value of DOA estimation error in logarithm vs. DOA and array aperture angle $\beta$ (VA with $M=20$ and $d=\lambda / 4$, one source with uniform angular dispersion, $\left.\Delta_{0}=10^{\circ}\right)$ 


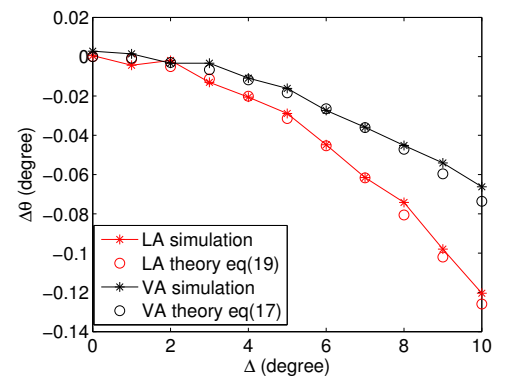

Fig. 4: DOA estimation error vs. the source angular dispersion $\Delta$ (LA with $M=20$ and $d=\lambda / 4$, VA with $M=20$ and $d=\lambda / 4$ and $\beta=100^{\circ}$, one source with uniform angular dispersion with $\theta=45^{\circ}$ )

In Figure 2, we compare the performance of MUSIC with the UCA and that with the LA versus the DOA separation, in the scenario of two sources. The DOA separation varies with $\theta_{c}=\frac{1}{2}\left(\theta_{1}+\theta_{2}\right)$ fixed to $60^{\circ}$, assuming that $\theta_{2}>\theta_{1}$. We can see that when the two sources are too close to each other, the estimation error can not be ignored despite the array geometry, due to the interference between the sources. For LA, $\Delta \theta_{1}$ decreases as the DOA separation increases and as $\theta_{1}$ tends to zero, by contrast, $\Delta \theta_{2}$ decreases as the interference phenomenon fades, but increases again along with the DOA separation, which coincides with the well-known conclusion that the ULA has a better performance for broadside sources. For UCA, both $\Delta \theta_{1}$ and $\Delta \theta_{2}$ tend to zero quickly as the DOA separation increases, which is in adequacy with the scenario of one source where the DOA estimation error is null and outperforms its LA partner. In addition, it is interesting to see the validity of the approximated expression (25) and (26) for describing the trend and the sign of the estimation error when the sources are close to each other.

In Figure 3, the absolute value in logarithm of the estimation error of one uniform distributed source with $\Delta_{0}=10^{\circ}$ is plotted with respect to the DOA and the array aperture angle $\beta=\pi-2 \alpha$, to validate the approximated expression (17). We note $\beta_{\text {min }}$ for the array aperture angle corresponding to the minimum value of $|\Delta \theta|$, and $\beta_{\text {min }}$ is plotted versus $\theta$ in the dashed line. According to (18), to have a null estimation error, $\beta \approx 49.7^{\circ}$ regardless of the value of DOA, which corresponds to the simulation result illustrated in Figure $3 \mathrm{~b}$.

In Figure 4, the DOA estimation error of MUSIC versus the angular dispersion of the signal source with $\theta=45^{\circ}$ are presented, in the case of LA and VA with $\beta=100^{\circ}$. We can observe the validation of expression (17) and (19) which makes it possible to consider the DOA estimation error as an explicit convex quadratic function of the angular dispersion of the signal source. Furthermore, regardless of a more complicated geometry, VA outperforms LA, which reveals the interest to optimize the array geometry to have a better performance.

\section{CONCLUSION}

In this paper, we have investigated the impact of the array geometry on the performance of the MUSIC-based DOA estimation in the presence of the spatially distributed sources. We have found that the DOA estimation error can be reduced and even canceled for particular array geometries in the case of one source or in the case where the DOA separation between two sources is large enough. Simulations which are carried out are in adequacy with the proposed theoretical results.

\section{REFERENCES}

[1] S.Valaee, B.Champagne, and P.Kabal, "Parametric localization of distributed sources," IEEE Trans. on Signal Processing, vol. 43, no. 9, pp. 2144-2153, 1995.

[2] H. Krim and M. Viberg, "Two decades of array signal processing research: the parametric approach," Signal Processing Magazine, IEEE, vol. 13, no. 4, pp. 67-94, 1996.

[3] P. Stoica and N. Arye, "Music, maximum likelihood, and cramer-rao bound," Acoustics, Speech and Signal Processing, IEEE Trans. on, vol. 37, no. 5, pp. 720-741, 1989.

[4] N. Chu, A.. Djafari, and J. Picheral, "Robust Bayesian super-resolution approach via sparsity enforcing a priori for near-field aeroacoustic source imaging," Journal of Sound and Vibration, vol. 332, no. 18, pp. 43694389, 2013.

[5] D. Astély and B. Ottersten, "The effects of local scattering on direction of arrival estimation with music," Signal Processing, IEEE Trans. on, vol. 47, no. 12, pp. 3220-3234, 1999.

[6] W.Xiong, J.Picheral, and S.Marcos, "Performance analysis of MUSIC in the presence of model errors due to the spatial distributions of sources," in ICASSP, 2015.

[7] J. Abou Chaaya, J. Picheral, and S. Marcos, "Localization of spatially distributed near-field sources with unknown angular spread shape," Signal Processing, vol. 106, pp. 259-265, 2015.

[8] H. Gazzah and K. Abed-Meraim, "Optimum ambiguity-free directional and omnidirectional planar antenna arrays for doa estimation," Signal Processing, IEEE Trans. on, vol. 57, no. 10, pp. 3942-3953, 2009.

[9] H. Gazzah and S. Marcos, "Cramer-rao bounds for antenna array design," Signal Processing, IEEE Trans. on, vol. 54, no. 1, pp. 336-345, 2006.

[10] J. Shin, Y. Lee, and H. Kim, "Reduced-complexity maximum likelihood direction-of-arrival estimation based on spatial aliasing," Signal Processing, IEEE Trans. on, vol. 62, no. 24, pp. 6568-6581, 2014. 